\title{
Identificação de casos psiquiátricos em estudos epidemiológicos multifásicos: métodos, problemas e aplicabilidade
} Psychiatric cases identification by multi steps epidemiological studies: methods, problems and applicability

Sérgio B Andreolia, Naomar de Almeida Filhob, Evandro SF Coutinhoc e Jair de J Maria $^{\mathrm{a}}$

aD epartamento de Psiquiatria da U niversidade Federal de São Paulo. São Paulo, SP, Brasil. 'Instituto de Saúde Coletiva da U niversidade Federal da Bahia. Salvador, BA, Brasil. 'D epartamento de Epidemiologia da Escola Nacional de Saúde Pública. Rio de Janeiro, RJ, Brasil 


\title{
Identificação de casos psiquiátricos em estudos epidemiológicos multifásicos: métodos, problemas e aplicabilidade* Psychiatric cases identification by multi steps epidemiological studies: methods, problems and applicability
}

\author{
Sérgio B Andreolia, Naomar de Almeida Filhob, Evandro SF Coutinhoc e Jair de J Maria \\ aD epartamento de Psiquiatria da U niversidade Federal de São Paulo. São Paulo, SP, Brasil. 'Instituto \\ de Saúde Coletiva da U niversidade Federal da Bahia. Salvador, BA, Brasil. 'D epartamento de Epidemi- \\ ologia da Escola Nacional de Saúde Pública. Rio de Janeiro, RJ, Brasil
}

\section{Descritores}

Transtornos mentais, epidemiologia\#.

Escalas de gradação psiquiátrica\# ${ }^{\#}$.

Estudos multicêntricos, métodos".

Inquéritos de morbidade\#.

Prevalência. Estudos

epidemiológicos. Entrevista

psiquiátrica padronizada. - Estudos de avaliação.

\section{Resumo}

\section{Objetivo}

Discutir aspectos metodológicos da estratégia de identificação de casos psiquiátricos, em duas etapas, em estudos epidemiológicos.

\section{Métodos}

Analisa-se a metodologia utilizada no Estudo Multicêntrico de Morbidade Psiquiátrica do Adulto realizado em três cidades brasileiras, entre 1990-1991. Na primeira etapa do citado estudo, uma amostra aleatória (6.740 indivíduos) da população foi selecionada e submetida a um rastreamento com o Questionário de Morbidade Psiquiátrica do Adulto - QMPA. Na segunda etapa, uma subamostra (775 indivíduos) foi selecionada e submetida ao Inventário de Sintomas do DSMIII (Diagnostic and Statistical Manual of Mental Disorders), aplicado por psiquiatras e psicólogos treinados, para confirmação-diagnóstica.

Resultados

São descritos os procedimentos empregados para a estimativa das prevalências, mostrando que o fraco desempenho da escala de rastreamento não compromete o método.

Conclusão

A vantagem da metodologia é a de corrigir as distorções apresentadas pelos instrumentos atuais de identificação de casos psiquiátricos.

\author{
Abstract \\ Objective \\ To discuss methodological aspects of the two stages in the identification of psychiatric \\ cases in epidemiological studies. \\ Methods \\ Analyze the methodology used in the Multicentric Psychiatric Morbidity Study, which \\ was conducted in three Brazilian cities (São Paulo, Brasília and Porto Alegre). In \\ the first stage of that study, a random sample (6,740 individuals) of the population
}

Correspondência para/Correspondence to: Sérgio Baxter Andreoli

Núcleo de Estatística e Metodologia Aplicadas Rua Dr. Bacelar, 334, Vila Clementino

04023-900 São Paulo, SP, Brasil

E-mail: andreoli@psiquiatria.epm.br
*Estudo financiado pela Fapesp (Processo no 94/0526-0).

Edição subvencionada pela Fapesp (Processo no 00/01601-8)

Recebido em 17/6/1999. Reapresentado em 19/4/2000. Aprovado em 4/5/2000. 
was drawn and all the participants were screened with the Questionnaire of Psychiatric Morbidity of the Adult (QMPA). In the second stage, a sample (775 individuals) of this population was drawn and these individuals were submitted to the Inventory of Symptoms of DSM-III, carried out by psychiatrists and trained psychologists.

\section{Results}

The study procedure for estimating the prevalence is described in details, showing that though the screening scales are a weak tool, they don't interfere with the methodology.

Conclusion

The advantage of this methodology is to correct any distortions caused by the current tools used in the identification of psychiatric cases.

\section{INTRO DUÇÃO}

O objetivo do presente estudo é avaliar a aplicabilidade dos métodos de identificação de casos psiquiátricos, em amostras populacionais, utilizando procedimentos de rastreamento, com base nos procedimentos e resultados da análise dos dados do Estudo Multicêntrico de Morbidade Psiquiátrica do Adulto, ${ }^{1}$ conduzido em três cidades brasileiras, entre 1990-1991. Nesse sentido, são apresentados, em detalhe, as etapas e os procedimentos empregados no citado estudo para as estimativas de prevalência, procurando demonstrar que o desempenho de escalas de rastreamento, caso corretamente avaliado em termos de sensibilidade, especificidade e valor preditivo, não compromete necessariamente a exeqüibilidade do método.

Os inquéritos populacionais são estudos epidemiológicos que se baseiam em um número grande de entrevistas domiciliares. Em estudos de morbidade psiquiátrica, a entrevista-diagnóstica realizada por psiquiatras é pouco indicada por se tratar de procedimento caro e de reduzida confiabilidade. A utilização de procedimentos padronizados de identificação de caso, portanto, constitui uma forma de baratear os custos e, assim, viabilizar tais estudos, aumentando sua confiabilidade. A década de 70 foi marcada pelo desenvolvimento de procedimentos dessa natureza, resultando na construção de série de instrumentos padronizados, destinados a auxiliar tanto o diagnóstico psiquiátrico na clínica como a identificação de casos em estudos epidemiológicos.

Os instrumentos padronizados podem ser classificados em entrevistas semi-estruturadas, entrevistas totalmente estruturadas e escalas de rastreamento. As entrevistas semi-estruturadas são compostas por listas de sintomas psiquiátricos elaboradas a partir de observações de pacientes da clínica; em geral, são desenhadas para serem utilizadas por clínicos habilitados. As entrevistas totalmente estruturadas também são compostas por listas de sintomas psiquiátricos, porém a presença ou a ausência do sintoma é estabelecida por intermédio de uma árvore de decisões totalmente estruturada; são preferencialmente administradas por entrevistadores leigos.

As escalas de rastreamento foram os primeiros instrumentos padronizados utilizados nos levantamentos epidemiológicos. Contêm questões que se referem a queixas psicológicas, psicossomáticas e fisiológicas encontradas nos transtornos psiquiátricos e podem ser auto-aplicadas ou aplicadas por entrevistadores leigos. Ao indivíduo é atribuído um escore total, resultado da soma das respostas positivas. Para cada escala, calcula-se um ponto de corte que melhor discrimina os indivíduos "doentes" dos "não-doentes".

Até o início da década de 70, existia uma pequena família dessas escalas breves de rastreamento, muito parecidas no conteúdo, que foram utilizadas em aproximadamente um terço dos estudos epidemiológicos realizados no período para medir construtos como: "saúde mental", "transtorno psiquiátrico", "doença mental", "ajuste emocional", "sintomas de estresse" e "sintomas psicofisiológicos". Não existia, nessa época, uma preocupação dos pesquisadores com uma melhor discriminação dos casos identificados, o que tornava os primeiros estudos epidemiológicos muito genéricos quanto ao diagnóstico de doença mental. ${ }^{14}$ As taxas de prevalência de doença mental eram calculadas tomando como base o escore do instrumento que melhor discriminava casos de não-casos.

A partir da década de 70, houve uma mudança na posição dos psiquiatras com relação à importância do diagnóstico, levando, por exemplo, ao desenvolvimento de sistemas classificatórios multiaxiais como o DSM-III. ${ }^{2}$ A partir daí, o esforço de desenvolvimento de instrumentos de identificação de casos concentrouse em métodos capazes de melhor discriminar o diagnóstico psiquiátrico. As escalas de rastreamento, den- 
tro desse contexto, passaram a ser desenvolvidas e utilizadas como indicadoras de casos potenciais e não como instrumento diagnóstico individual. A aplicação desse tipo de instrumento em uma primeira etapa de pesquisa, em conjunto com instrumentos estruturados, dentro de um levantamento epidemiológico, caracteriza a metodologia dos chamados desenhos multifásicos.

Levantamento populacional conduzido com esse método oferece vantagens consideráveis: apresenta um custo relativamente baixo em um tempo curto, pois a grande maioria das entrevistas é feita por entrevistadores leigos, com um instrumento de fácil aplicação, e apenas uma proporção pequena de casos precisam ser submetidos a entrevista com psiquiatra. É possível um treinamento eficiente de clínicos na aplicação da entrevista padronizada, e a natureza diagnóstica, bem como a quantidade de transtornos específicos de morbidade, podem ser determinados dentro de limites de confiança aceitáveis. ${ }^{9}$ Muitos levantamentos populacionais têm sido conduzidos utilizando estratégia similar para a identificação de casos, sendo o estudo brasileiro um exemplo.

\section{MÉTODOS}

\section{Aspectos do estudo multicêntrico}

O Estudo Multicêntrico de Morbidade Psiquiátri$\mathrm{ca}^{1}$ é o primeiro estudo epidemiológico de transtornos psiquiátricos realizado no Brasil que utiliza metodologia de identificação de caso em duas etapas, com instrumentos padronizados. O objetivo desse estudo foi estimar a prevalência de morbidade psiquiátrica em amostras representativas da população acima de 15 anos, residentes em três áreas metropolitanas brasileiras (Brasília, São Paulo e Porto Alegre). Para a identificação de casos psiquiátricos, foram utilizadas duas escalas psiquiátricas aplicadas em duas etapas (Figura). Na primeira etapa, o Questionário de Morbidade Psiquiátrica do Adulto (QMPA) foi aplicado para rastreamento de casos psiquiátricos; esses indivíduos compõem o que passará a chamar de amostra. Na segunda fase, $30 \%$ de prováveis casos e $10 \%$ de prováveis não-casos, identificados pelo QMPA, foram selecionados e submetidos ao inventário de sintomas do DSM-III; esse subgrupo de indivíduos compõe o que passará a chamar de subamostra.

\section{Plano amostral}

A amostragem realizada em Brasília foi estratificada geograficamente, tendo como estratos o plano piloto e as cidades satélites. De acordo com a distribuição demográfica, os estratos foram subdivididos em

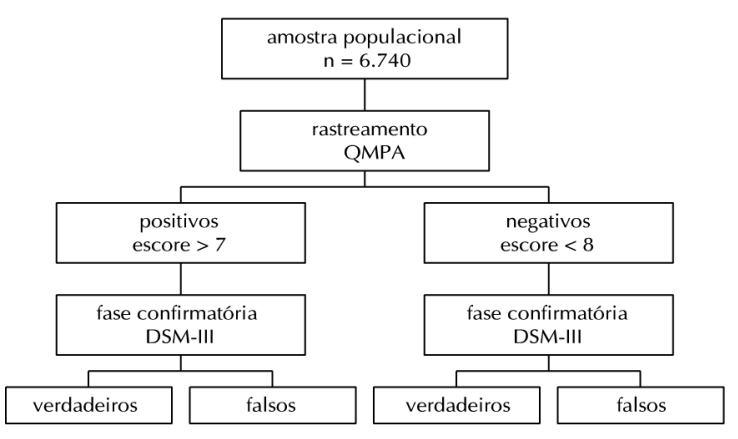

Figura - Levantamento populacional com rastreamento.

setores, segundo a situação socioeconômica da população. Dentro de cada setor, o tamanho da amostra de domicílios foi proporcional ao tamanho da sua população. Com base em um mapeamento atualizado, foi sorteado um domicílio como ponto de partida para cada entrevistador, que prosseguia incluindo na amostra a residência de número imediatamente superior, até esgotar o número de questionários previamente determinado pelos supervisores de campo. O tamanho da amostra foi calculado em 741 residências, tendo como base a média de 2,7 pessoas maiores de 15 anos por domicílio em Brasília. No total foram obtidas informações de 2.347 sujeitos.

Em São Paulo, a amostragem foi estratificada por condição socioeconômica. A cidade é composta por 48 distritos, dos quais 3 foram selecionados com base nos indicadores de saúde como representativos de toda a população. Assim, foram selecionados os distritos de Brasilândia, Vila Guilherme e Aclimação para as categorias socioeconômicas inferior, média e superior. A amostragem foi composta por $56,1 \%$ da população de Brasilândia (146 setores censitários), 27,2\% de Vila Guilherme (65 setores censitários) e 16,7\% da Aclimação (63 setores censitários). Foram selecionados 30 setores censitários para sorteio de 20 domicílios por setor (17 de Brasilândia, 8 de Vila Guilherme e 5 de Aclimação). Foram substituídas 93 famílias (15,5\% da amostra): 33 na Brasilândia (6 recusas e 27 ausências), 28 na Vila Guilherme (4 recusas e 24 ausências) e 32 na Aclimação (16 recusas e 16 ausentes). A amostra efetivamente pesquisada foi de 620 residências, totalizando uma amostra de 1.742 sujeitos maiores de 15 anos.

Em Porto Alegre, a Fundação Metropolitana de Desenvolvimento (Metroplan) identifica 96 zonas de tráfego (ZT). Cada ZT engloba 740 setores censitários, com uma média de 350 unidades habitacionais e 1.750 habitantes em cada setor. Cada ZT foi ordenada, segundo a renda média em 1986, em 10 estratos socioeconômicos. De cada um dos estratos foram sorteados 3 domicílios localizados na 
lista de domicílios por setor, atualizada para o censo de 1991. Cada um desses domicílios foi o identificador do setor censitário que passou a ser o conglomerado da investigação. Dentro de cada um desses setores censitários foram sorteados 37 domicílios, sendo 30 para a amostra e 7 para eventuais reposições. A amostra efetivamente pesquisada foi de 998 residências, com 903 famílias concordando em participar do estudo, o que resultou numa perda de $9,5 \%$. No total foram obtidas as informações de 2.384 sujeitos.

\section{Instrumentos}

Questionário de Morbidade Psiquiátrica do Adulto (QMPA). Trata-se de uma escala de rastreamento desenvolvida no Brasil por Santana ${ }^{13}$ com 45 questões, na sua última versão. As questões do QMPA abrangem os mais freqüentes sinais e sintomas característicos das doenças mentais, além de questões sobre tratamento psiquiátrico, uso anterior e atual de drogas psicofarmacológicas. O QMPA pode ser auto-aplicado ou aplicado por entrevistador leigo e pode ser respondido por terceiros. As alternativas de respostas são sim ou não, às quais são atribuídos valores 1 ou 0 . As primeiras 43 questões devem ser respondidas sobre o próprio indivíduo do qual se quer saber o estado de saúde mental, e as duas últimas questões (questões 44 e 45) referem-se à sintomatologia apresentada por qualquer um dos membros da família do indivíduo do qual se quer saber o estado de saúde mental.

Santana ${ }^{13}$ validou o QMPA realizando um estudo-piloto e um teste de campo. Em ambos, os escores obtidos pelo QMPA foram confrontados com uma entrevista psiquiátrica semi-estruturada utilizada para a definição de caso, tendo como critério diagnóstico a Classificação Internacional de Doenças - versão 8 (CID-8). O QMPA, para o ponto de corte $6 / 7$, apresentou uma sensibilidade de $75 \%$ no estudo-piloto e $93 \%$ no teste de campo e uma especificidade de $53 \%$ e $94 \%$. Estudou, também, a confiabilidade do QMPA que apresentou um Kappa ponderado de 0,88 .

Inventário de Sintomas do DSM-III. O inventário foi desenvolvido no Departamento de Psiquiatria da Universidade de Washington, St. Louis, EUA, para verificar os diagnósticos gerados pelo Diagnostic Interview Schedule (DIS). ${ }^{12}$ Investiga 39 diagnósticos do DSM-III ${ }^{2}$ e pode funcionar como um padrão válido para a avaliação de sintomas psiquiátricos e, conseqüentemente, identificação de caso psiquiátrico, desde que aplicado por clínicos treinados e familiarizados com os critérios do DSM-
III. ${ }^{8}$ Todos os diagnósticos que aparecem no instrumento são definidos e têm seus critérios estabelecidos no DSM-III. Para alguns dos diagnósticos, o instrumento apresenta uma lista de sintomas, e para ser classificado como positivo em um ou mais de um diagnóstico, o indivíduo deve apresentar um número mínimo de sintomas e preencher critérios definidos no DSM-III, tais como: pertencer a uma dada faixa de idade determinada, ter apresentado tais sintomas durante um período de tempo determinado, não apresentar outro diagnóstico que exclua a sua presença ou qualquer outra condição de exclusão.

Para a codificação dos sintomas são utilizados 3 níveis: (1) o sintoma está ausente ou não há evidência clínica de ser relevante; (2) o sintoma está presente e é relevante do ponto de vista clínico psiquiátrico; (3) incerteza na distinção entre presença e ausência do sintoma. Para a codificação-diagnóstica são utilizados 4 níveis: (1) ausente; (2) preenche os critérios do DSM-III e não é excluído por outro diagnóstico do DSM-III; (3) preenche os critérios do DSM-III somente se as regras de exclusão forem ignoradas; (4) incerto. Todos os diagnósticos são feitos tomando como base o tempo de vida do entrevistado; assim, para os indivíduos que preenchem os critérios para um diagnóstico, o período de aparecimento desse é codificado em: (1) nas últimas duas semanas; (2) de duas a menos de um mês; (3) de um mês a 6 meses; (4) de 6 meses a um ano; (5) menos de um ano (não sabe quando); (6) mais de um ano atrás.

O inventário de sintomas do DSM-III está organizado para cada diagnóstico de formas diferentes. Existem diagnósticos sem lista e com lista de sintomas e critérios, sendo que esses últimos podem apresentar a lista de sintomas hierarquizada ou não. A hierarquização, nesse caso, significa que um ou mais sintomas devem ser preenchidos de modo afirmativo para que um grupo subseqüente de sintomas seja investigado. Quando não hierarquizado, o entrevistador deve investigar todos os sintomas do diagnóstico. No caso dos diagnósticos sem lista de sintomas e critérios, o entrevistador deve indicar o código diagnóstico de acordo com os critérios da classificação-diagnóstica do DSM-III.

Para o caso em que o indivíduo preenche os critérios para um diagnóstico e o período de aparecimento foi no último ano (codificação de tempo 1 ou 2), o entrevistado deve responder se esteve incomodado por esses sintomas a maior parte do tempo: (1) não; (2) sim. Esse indicador, originalmente ausente do inventário de sintomas do DSM-III, foi introduzido para uma avaliação da gravidade do quadro apresentado pelo paciente. 


\section{Procedimentos do estudo de campo}

Sorteados os domicílios, as famílias foram procuradas pelos entrevistadores, em sua maioria estudantes de medicina ou de outras profissões de saúde, previamente treinados para aplicar o QMPA. Quando existia dúvida se o endereço sorteado havia se alterado (por exemplo: em caso de demolição ou situação de abandono) eram feitas até 3 tentativas pelos entrevistadores antes das famílias serem substituídas. No domicílio, o entrevistador solicitava a presença da dona da casa ou de pessoa adulta e adequada a prestar as informações (informante-chave). Esse informante-chave respondia às questões do QMPA, também para os outros membros da família, maiores de 15 anos.

Com um intervalo que variou entre um e quatro semanas da primeira etapa do estudo, uma subamostra de indivíduos foi entrevistada por meio do inventário de sintomas do DSM-III. O estudo empregou um total de 25 psiquiatras e psicólogos com treinamento clínico para a condução da fase de confirmação diagnóstica. Esses profissionais foram treinados para aplicar o inventário de sintomas do DSM-III por meio de cursos sobre os critérios diagnósticos, vídeo-tapes de entrevistas psiquiátricas e entrevistas supervisionadas. Os resultados do exame de confirmação diagnóstica eram registrados de acordo com o nível de certeza da presença de patologia, duração do transtorno e grau de gravidade. A gravidade foi avaliada pelos próprios entrevistados, nos casos em que o entrevistado havia preenchido os critérios para qualquer um dos diagnósticos no último mês.

\section{Técnica de cálculo das estimativas de prevalência}

\section{Estimativas globais}

Estimativas globais de prevalência foram calculadas para toda a vida dos indivíduos (lifetime prevalence). Os cálculos foram realizados pela adequação da capacidade de identificação de caso do QMPA, tomando como padrão o diagnóstico feito por meio do inventário de sintomas do DSM-III. Para tanto, foram calculados, na subamostra, os in- dicadores de validade do QMPA (sensibilidade, especificidade, valor preditivo positivo e valor preditivo negativo), com o ponto de corte $7 / 8$, utilizando como padrão de comparação a subamostra com qualquer dos 39 diagnósticos do DSM-III, excetuando tabagismo.

Na Tabela 1, e conforme já analisado em outra publicação, ${ }^{5}$ o desempenho do QMPA mostrou-se abaixo do observado em estudos de validação anteriores, com proporções de classificação incorreta acima de $33 \%$ em todas as subamostras. Os valores de sensibilidade ficaram em torno de $50 \%$ e os valores de especificidade variaram de $70 \%$ a $80 \%$, mostrando um desempenho na direção contrária ao esperado para um instrumento de rastreamento, qual seja, o aumento da proporção de casos na subamostra.

Os valores preditivos positivos foram diferentes entre as cidades, apresentando-se adequados nas cidades de Brasília (83\%) e Porto Alegre (70\%), porém reduzidos na cidade de São Paulo (abaixo de 50\%). O valor preditivo negativo, ao contrário, apresentou valores insatisfatórios nas cidades de Brasília (53\%) e Porto Alegre (62\%), sendo mais adequado em São Paulo (72\%).

O valor preditivo positivo foi utilizado para as estimativas de prevalência global na amostra, pois permite calcular a proporção de indivíduos positivos no QMPA que possuem algum diagnóstico do DSMIII. Essas estimativas foram corrigidas pelo inverso do valor preditivo negativo, segundo a equação de Fleiss, ${ }^{7}$ em que Pe é a estimativa de prevalência, ps é a proporção de supostos casos identificados pelo QMPA na amostra, pn é a proporção de não suspeitos, VP+ e VP- são os valores preditivos do QMPA, conforme segue:

$$
\mathrm{Pe}=\mathrm{ps}(\mathrm{VP}+)+\mathrm{pn}(1-\mathrm{VP}-)
$$

O erro-padrão (EP) das estimativas de prevalência globais foram calculadas de acordo com a seguinte equação:

$$
E P(P e)=\sqrt{\frac{P e(1-P e)}{N}\left(1+(1-K) \frac{N-n}{n}\right.},
$$

Tabela 1 - Indicadores de desempenho do QMPA com o inventário de sintomas do DSM-III, distribuídos por gênero em três capitais brasileiras. Brasil, 1991.

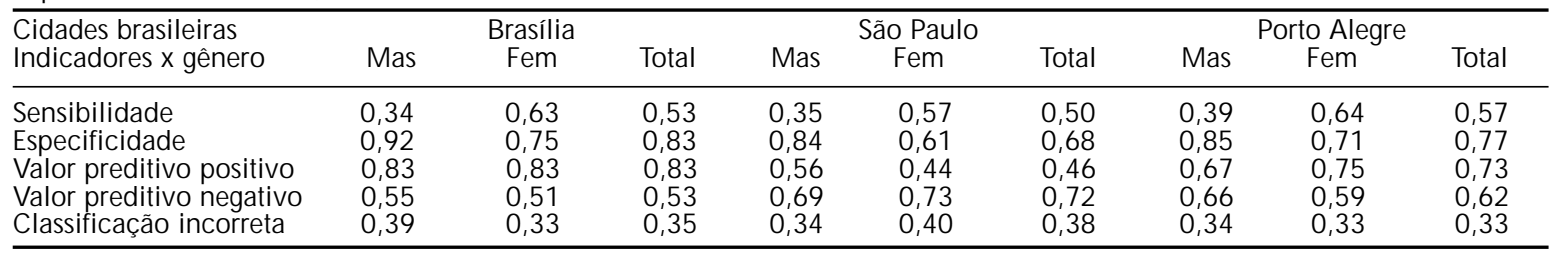

Q MPA - Q uestionário de Morbidade Psiquiátrica do Adulto DSM -III (Diagnostic and Statistical Manual of M ental Disorders) 
onde

$$
K=\frac{1-p s}{p s} \times \frac{(P e-(1-V P-))}{P e(1-P e)},
$$

em que Pe é a estimativa global de prevalência, ps é a prevalência de supostos casos na amostra, N é o número de indivíduos entrevistados na amostra, $\mathrm{n}$ é o número de indivíduos entrevistados na subamostra e VP - é o valor preditivo negativo.

Os resultados dos cálculos das estimativas de prevalência globais são mostrados na Tabela 2, em que se pode observar as proporções de supostos casos identificados pelo QMPA na amostra. As proporções apresentam-se com valores maiores para as mulheres, com aproximadamente o dobro daqueles apresentados pelos homens, nas três cidades estudadas. A cidade de São Paulo, em termos absolutos, apresenta as proporções maiores de casos supostos, entretanto, após a correção pelo desempenho do QMPA, a estimativa de prevalência global é menor do que aquelas apresentadas nas outras cidades.

A Tabela 3 mostra os parâmetros para os cálculos do erro-padrão, cuja estimativa de prevalência global foi de aproximadamente $0,03 \%$ para as três cidades. Quando distribuído por gênero, entretanto, as estimativas variaram de $0,04 \%$ a $0,06 \%$, com valores maiores para as mulheres nas cidades de São Paulo e Porto Alegre.

\section{Estimativas por diagnóstico}

As estimativas de prevalência por grupo-diagnóstico foram calculadas considerando as proporções de cada grupo-diagnóstico na subamostra de casos confirmados, multiplicadas pela prevalência global, de acordo com a seguinte fórmula:

$$
\mathrm{Pd}=\mathrm{Pe}(\mathrm{Fd} / \mathrm{Tc})
$$

em que Pd é a estimativa de prevalência do grupodiagnóstico, Pe a estimativa de prevalência global (Tabela 2), Fd é a frequiência de casos confirmados do diagnóstico e Tc corresponde ao total de indivíduos confirmados como tendo pelo menos um diagnóstico do DSM-III, identificados na segunda etapa do estudo.

A Tabela 4 mostra as freqüências de cada grupodiagnóstico de acordo com gênero e cidade, e observa-se que a soma das freqüências é maior do que o número de indivíduos, isto porque, segundo a metodologia adotada, os indivíduos podiam ter mais do que um diagnóstico. A Tabela 5 mostra os resultados da aplicação da fórmula acima para o cálculo das estimativas de prevalência por grupo-diagnóstico.

\section{DISCUSSÃO METODOLÓGICA}

A identificação de casos psiquiátricos em estudos epidemiológicos, envolvendo uma amostragem randômica da população, necessita de uma etapa de rastreamento antes do exame psiquiátrico. No exemplo do estudo multicêntrico brasileiro, esse procedimento não é simples por causa do desempenho insatisfatório das escalas de rastreamento. ${ }^{5}$ Mesmo assim, é possível calcular as estimativas de prevalência e seus erros-padrão sem prejuízo para a comparação dos resultados, tanto entre cidades estudadas quanto com os resultados de levantamentos populacionais que empregaram metodologia similar. ${ }^{1}$

As razões que melhor justificam a utilização de técnicas de rastreamento para a identificação de casos

Tabela 2 - Cálculo das estimativas de prevalência globais, utilizando as proporções de indivíduos supostos; casos identificados pelo Q M PA (>7) na amostra da população de três cidades brasileiras $(n=6.740)$, balanceadas pelos valores preditivos do Q M PA

\begin{tabular}{|c|c|c|c|c|c|c|c|c|c|}
\hline \multirow{2}{*}{$\begin{array}{l}\text { Cidades brasileiras } \\
\text { Parâmetros para o cálculo da prevalência }\end{array}$} & \multicolumn{3}{|c|}{ Brasília } & \multicolumn{3}{|c|}{ São Paulo } & \multicolumn{3}{|c|}{ Porto Alegre } \\
\hline & Masc & Fem & Total & Masc & Fem & Total & Masc & Fem & Total \\
\hline $\begin{array}{l}\text { Supostos casos } \\
\text { Supostos não casos } \\
\text { Valor preditivo positivo } \\
\text { Valor preditivo negativo }\end{array}$ & $\begin{array}{l}0,07 \\
0,93 \\
0,83 \\
0,55\end{array}$ & $\begin{array}{l}0,16 \\
0,84 \\
0,83 \\
0,51\end{array}$ & $\begin{array}{l}0,12 \\
0,88 \\
0,83 \\
0,53\end{array}$ & $\begin{array}{l}0,12 \\
0,88 \\
0,56 \\
0,69\end{array}$ & $\begin{array}{l}0,26 \\
0,75 \\
0,44 \\
0,73\end{array}$ & $\begin{array}{l}0,18 \\
0,82 \\
0,46 \\
0,72\end{array}$ & $\begin{array}{l}0,07 \\
0,93 \\
0,67 \\
0,66\end{array}$ & $\begin{array}{l}0,18 \\
0,81 \\
0,75 \\
0,59\end{array}$ & $\begin{array}{l}0,13 \\
0,87 \\
0,73 \\
0,62\end{array}$ \\
\hline Estimativa de prevalência global & 0,47 & 0,54 & 0,51 & 0,34 & 0,31 & 0,31 & 0,36 & 0,47 & 0,43 \\
\hline
\end{tabular}
distribuídos por gênero. Brasil, 1991.

Tabela 3 - Cálculo dos desvios-padrão das estimativas de prevalência globais para as três cidades brasileiras distribuídos por

\begin{tabular}{|c|c|c|c|c|c|c|c|c|c|}
\hline \multirow{2}{*}{$\begin{array}{l}\text { Cidades brasileiras } \\
\text { Parâmetros para o cálculo de erro-padrão }\end{array}$} & \multirow{2}{*}{\multicolumn{2}{|c|}{$\begin{array}{l}\text { Brasília } \\
\text { Fem }\end{array}$}} & \multirow[b]{2}{*}{ Total } & \multirow{2}{*}{\multicolumn{2}{|c|}{$\begin{array}{l}\text { São Paulo } \\
\text { Fem }\end{array}$}} & \multirow[b]{2}{*}{ Total } & \multicolumn{3}{|c|}{ Porto Alegre } \\
\hline & & & & & & & Masc & Fem & total \\
\hline $\begin{array}{l}\text { Estimativa de prevalência global } \\
\text { Supostos casos } \\
\text { Valor preditivo negativo } \\
\text { Número de indivíduos na amostra } \\
\text { Número de indivíduos na subamostra }\end{array}$ & $\begin{array}{l}0,47 \\
0,07 \\
0,55 \\
1.086 \\
106\end{array}$ & $\begin{array}{c}0,54 \\
0,16 \\
0,51 \\
1.258 \\
168\end{array}$ & $\begin{array}{l}0,51 \\
0,12 \\
0,53 \\
2.344 \\
274\end{array}$ & $\begin{array}{l}0,34 \\
0,12 \\
0,69 \\
845 \\
71\end{array}$ & $\begin{array}{l}0,31 \\
0,26 \\
0,73 \\
897 \\
151\end{array}$ & $\begin{array}{c}0,31 \\
0,18 \\
0,72 \\
1.742 \\
222\end{array}$ & $\begin{array}{l}0,36 \\
0,07 \\
0,66 \\
1.075 \\
97\end{array}$ & $\begin{array}{c}0,47 \\
0,18 \\
0,59 \\
1.309 \\
182\end{array}$ & $\begin{array}{c}0,43 \\
0,13 \\
0,62 \\
2.384 \\
279\end{array}$ \\
\hline Erro-padrão & 0,04 & 0,04 & 0,03 & 0,06 & 0,03 & 0,03 & 0,05 & 0,04 & 0,03 \\
\hline
\end{tabular}
gênero. Brasil, 1991. 
Tabela 4 - Freqüência dos grupos-diagnóstico na subamostra de três cidades brasileiras distribuídos por gênero. Brasil, 1991.

\begin{tabular}{|c|c|c|c|c|c|c|}
\hline \multirow[b]{2}{*}{ Diagnósticos do DSM-III } & \multicolumn{2}{|c|}{ Brasília } & \multicolumn{2}{|c|}{ São Paulo } & \multicolumn{2}{|c|}{ Porto Alegre } \\
\hline & Masc & Fem & Masc & Fem & Masc & Fem \\
\hline Síndrome cerebrorgânica & 2 & 2 & 0 & 0 & 2 & 4 \\
\hline Abuso e dependência de álcool & 18 & 2 & 12 & 0 & 19 & 5 \\
\hline Abuso e dependência de drogas & 0 & 1 & 1 & 0 & 3 & 7 \\
\hline Esquizofrenias & 0 & 0 & 0 & 1 & 2 & 2 \\
\hline Transtornos afetivos - depressão & 3 & 8 & 0 & 7 & 7 & 30 \\
\hline Transtornos afetivos - mania e ciclotimias & 1 & 0 & 0 & 1 & 2 & 1 \\
\hline Transtornos delirantes, psicóticos e outros & 0 & 1 & 0 & 2 & 2 & 3 \\
\hline Transtornos conversivos e somatodissociativos & 4 & $2 \overline{7}$ & 1 & 8 & 2 & 17 \\
\hline Transtornos de pânico, ansiedade e outros & 16 & 45 & 6 & 25 & 6 & 29 \\
\hline Transtornos obsessivos compulsivos & 1 & 1 & 0 & 0 & 2 & 5 \\
\hline Fobias - agora, social e simples & 13 & 47 & 4 & 19 & 9 & 43 \\
\hline Transtorno de ajustamento & 3 & 4 & 0 & 2 & 2 & 3 \\
\hline Fatores psicológicos que afetam o físico & 13 & 18 & 8 & 11 & 3 & 9 \\
\hline Retardo mental & 4 & 6 & 3 & 3 & 3 & 9 \\
\hline Número de diagnósticos positivos & 78 & 162 & 35 & 79 & 64 & 167 \\
\hline Número de individuos com diagnóstico positivo & 56 & 111 & 26 & 52 & 41 & 105 \\
\hline
\end{tabular}

Tabela 5 - Estimativas de prevalência por grupo-diagnóstico em três cidades brasileiras distribuídos por gênero. Brasil, 1991.

\begin{tabular}{|c|c|c|c|c|c|c|}
\hline \multirow[b]{2}{*}{ Diagnósticos do DSM-III } & \multicolumn{2}{|c|}{ Brasília } & \multicolumn{2}{|c|}{ São Paulo } & \multicolumn{2}{|c|}{ Porto Alegre } \\
\hline & Masc & Fem & Masc & Fem & Masc & Fem \\
\hline Síndrome cerebrorgânica & 1,9 & 1,1 & & - & 1,7 & 2,0 \\
\hline Abuso e dependência de álcool & 15,0 & 1,1 & 15,2 & - & 16,0 & 2,5 \\
\hline Abuso e dependência de drogas & - & 0,5 & 1,3 & - & 2,4 & 3,5 \\
\hline Esquizofrenias & - & 0,0 & - & 0,6 & 1,7 & 1,0 \\
\hline Transtornos afetivos - depressão & 1,9 & 3,8 & - & 3,8 & 5,9 & 14,5 \\
\hline Transtornos afetivos - mania e ciclotimias & 0,9 & - & - & 0,6 & 1,7 & 0,5 \\
\hline Transtornos delirantes, psicóticos e outros & - & 0,5 & - & 1,2 & 1,7 & 1,5 \\
\hline Transtornos conversivos e somatodissociativos & 3,3 & 13,0 & 1,3 & 4,3 & 1,7 & 8,0 \\
\hline Transtornos de pânico, ansiedade e outros & 13,6 & 21,6 & 7,6 & 13,9 & 5,2 & 14,0 \\
\hline Transtornos obsessivos compulsivos & 0,9 & 0,5 & 0,0 & - & 1,7 & 2,5 \\
\hline Fobias - agora, social e simples & 10,8 & 22,7 & 4,9 & 10,4 & 7,7 & 20,5 \\
\hline Transtorno de ajustamento & 1,9 & 2,2 & - & 1,2 & 1,7 & 1,5 \\
\hline Fatores psicológicos que afetam o físico & 10,8 & 8,6 & 10,2 & 6,1 & 2,4 & 4,5 \\
\hline Retardo mental & 3,3 & 2,7 & 3,6 & 1,7 & 2,4 & 4,5 \\
\hline
\end{tabular}

em inquéritos populacionais são as seguintes: controlar o erro de identificação das escalas ${ }^{7}$ e reduzir o tamanho da amostra para posterior investigação. No estudo em pauta, a intenção, ao utilizar esse método, foi inicialmente reduzir o tamanho da amostra e aumentar a proporção de casos, entrevistando um número maior de supostos casos na etapa de confirmação-diagnóstica. Com a aplicação desse procedimento, a subamostra ficou com o tamanho de 775 indivíduos, e as proporções de casos com pelo menos um diagnóstico do DSM-III foram de $61 \%$ na cidade de Brasília, 35\% em São Paulo e 52\% em Porto Alegre.

O controle do erro de identificação do QMPA pode ser ilustrado quando se comparam as prevalências estimadas por meio dos valores preditivos positivos sem correção (ps x VP+) e as prevalências estimadas após a correção. Sem a correção, a prevalência foi de 9,9\% em Brasília, 8,3 \% em São Paulo e 9,5\% em Porto Alegre; podendo-se observar que existe entre elas uma pequena variação. Entretanto, quando corrigidas pelos falsos negativos (1-VP-), os valores das estimativas aumentam e mostram grande variação entre as cidades: $51 \%$ em Brasília, 31\% em São Paulo e $43 \%$ em Porto Alegre.

Dessa forma, as variações dos valores das estimativas foram devidas às proporções elevadas de falsos negativos (1- VP-) do QMPA, quais sejam: 47\% em Brasília, 28\% em São Paulo e 38\% em Porto Alegre. É bem conhecido na literatura que o indicador mais importante e crítico para uma escala de rastreamento é a proporção de falsos negativos ${ }^{6} \mathrm{e}$, no presente exemplo, não só desempenhou papel importante de controle de erro, mas também funcionou como importante moderador do exame psiquiátrico.

Esse papel moderador do exame psiquiátrico pode ser observado quando são comparadas as prevalências encontradas nas subamostras $(61 \% ; 35 \% ; 52 \%)$ e as prevalências estimadas $(51 \% ; 31 \% ; 43 \%)$. Embora seja uma comparação de dados brutos, no caso da subamostra, é ilustrativo observar que a redução relativa nas proporções foi de $16 \%, 11 \%$ e $17 \%$, portanto, uma atenuação diferenciada entre as cidades.

A observação desses resultados provoca reflexão acerca da utilização de técnicas de rastreamento em estudos epidemiológicos no campo da psiquiatria. As escalas são para esses estudos mais do que rastreadoras de caso psiquiátrico; são um fator de correção do erro-diagnóstico, e essa seria a maior justificativa para sua utilização.

De um modo geral, a identificação de caso psiquiátrico na pesquisa epidemiológica é ainda precária, pois está totalmente baseada em sintomas. Por esse 
motivo, não existem razões para acreditar que algum instrumento que se proponha a substituir o exame clínico possa ser definitivo nessa função. As críticas aos estudos que utilizam a etapa de rastreamento baseiam-se no fraco desempenho dos instrumentos de rastreamento. É preciso, entretanto, lembrar que o desempenho é sempre avaliado tomando como referência outros instrumentos, que por sua vez também terão problemas de validade e confiabilidade. Observando os resultados, é possível supor que, se por um lado a escala de rastreamento deixa de identificar casos, o inventário de sintomas do DSM-III, aplicado por um especialista, tende a identificar casos a mais. Ao confrontar os dois instrumentos, e efetuadas as devidas correções, as estimativas de prevalência podem efetivamente servir de base para as comparações entre as cidades.

O instrumento diagnóstico utilizado na segunda etapa do estudo multicêntrico, analisado no presente trabalho, mostrou-se adequado, mas possui o inconveniente de precisar de clínicos treinados para sua aplicação. Atualmente dispõe-se de uma versão estruturada do CIDI $^{15}$ (Composite International Diagnostic Interview) que investiga diagnósticos psiquiátricos baseados na Classificação Internacional de Doenças, versão 10. Além de utilizar uma clas-

\section{REFERÊNCIAS}

1. Almeida-Filho N, Mari JJ, Coutinho E, França JF, Fernandes J, Andreoli SB et al. Brazilian multicentric study of psychiatric morbidity: methodological feature and prevalence estimates. Br J Psychiatry 1997;171:524-9.

2. American Psychiatric Association. Diagnostic and statistical manual of mental disorders. 3rd ed. Washington (DC): 1980.

3. Andreoli SB, Mari JJ, Blay SL, Almeida Filho $\mathrm{N}$ de, Coutinho E, França JF et al. Estrutura fatorial do questionário de morbidade psiquiátrica de adultos aplicado em amostras populacionais de cidades brasileiras. Rev Saúde Pública 1994;28:249-60.

4. Andreoli SB. Estudo das manifestações psicopatológicas na população brasileira [Tese]. São Paulo: U niversidade Federal de São Paulo; 1997.

5. Andreoli SB, Blay SL, Mari JJ. Escalas de rastreamento de psicopatologia. Rev Psiquiatr Clín 1998;25:229-32.

6. Deming WE. An essay on screening, or on two-phase sampling, applied to survey of a community. Int Stat Rev 1977;45:29-37. sificação de doença atualizada, o CIDI pode ser aplicado por entrevistadores leigos treinados.*

O rastreamento é um procedimento desejável em estudos populacionais no campo da psiquiatria, no entanto, alguns cuidados devem ser tomados ao se replicar esse estudo, principalmente no que diz respeito à escolha dos instrumentos. As escalas de rastreamento para esse fim disponíveis na língua portuguesa e com estudos de desempenho são: o QMPA,${ }^{13}$ o General Health Questionnaire $\left(\mathrm{GHQ}^{10}\right)$ e o Self Report Questionnaire $\left(S_{R Q}^{11}\right)$. Desses, o QMPA e o SRQ são semelhantes na sua forma e conteúdo, e o GHQ apresenta algumas diferenças no conteúdo, mas a principal distinção entre elas encontra-se na forma de medida dos sintomas (escalas de Likert no caso do GHQ). O QMPA, entretanto, apresenta um número maior de estudos de desempenho em amostras populacionais brasileiras, ${ }^{3,4,13}$ fornecendo subsídios para o cálculo da amostra submetida à segunda etapa e para ajustes na aplicação em estudos futuros.

\section{AGRADECIMENTOS}

A Ellis D' Arrigo Busnello, Josimar F França, Jéferson G Fernandes, pela coordenação regional do estudo muticêntrico, e a Cláudio T Miranda, Sérgio L Blay e Marcos T Ferraz pelas críticas e pelos comentários.

7. Fleiss JL. The control of misclassification error. In: Statistical methods for rates and proportions. 2nd ed. New York: John Wiley; 1981.

8. Helzer JE, Robins LN, M cEvoy LT, Spitznagel EL, Stoltzman RK, Famer A et al. A comparison of clinical and diagnostic interview schedule diagnoses. Physician reexamination of lay-interviewed cases in the general population. Arch Gen Psychiatr 1985;42:657-66.

9. Henderson S, D uncan-Jones P, Byrne DG, Scott R, Adcock S. Psychiatric disorder in Canberra. A standardized study of prevalence. Acta Psychiatr Scand 1979;60:355-74.

10. Mari JJ, Williams P. A comparison of the validity of two psychiatric screening questionnaires (GHQ -12 and SRQ -20) in Brazil, using Relative O perating Characteristic (ROC) analysis. Psychol Med 1985;15:651-9.

11. Mari JJ, Blay SL, lacoponi E. Reliability of the Brazilian version of the clinical interview schedule. Bol O ficina Sanit Panamer 1986;100:77-83. 
12. Robins LN, Helzer JE, Croughan J, Ratcliff KS. The NIMH Diagnostic Interview Schedule: its history, characteristics, and validity. Arch Gen Psychiatr 1981;38:381-9.

13. Santana W. Estudo epidemiológico das doenças mentais em bairro de Salvador. Salvador: Instituto de Saúde da Bahia; 1982 (Série de Estudos em Saúde).

14. Weissman M M. Epidemiology overview. In: Hales RE, Frances Al, editors. American Psychiatric Association Annual Review. $6^{\text {th }}$ ed. Washington (DC): American Psychiatric Association; 1987. p. 574-85.
15. Wittchen HU, Robins LN, Cottler LB, Sartorius N, Burke JD, Regier D et al. Cross-cultural feasibility, reliability and sources of variance of the Composite International Diagnostic Interview (CIDI). Br J Psychiatry 1991;159:645-53. 


\title{
Identificação de casos psiquiátricos em estudos epidemiológicos multifásicos: métodos, problemas e aplicabilidade* Psychiatric cases identification by multi steps epidemiological studies: methods, problems and applicability
}

\author{
Sérgio B Andreolia, Naomar de Almeida Filhob, Evandro SF Coutinhoc e Jair de J Maria \\ aD epartamento de Psiquiatria da U niversidade Federal de São Paulo. São Paulo, SP, Brasil. 'Instituto \\ de Saúde Coletiva da U niversidade Federal da Bahia. Salvador, BA, Brasil. 'D epartamento de Epidemi- \\ ologia da Escola Nacional de Saúde Pública. Rio de Janeiro, RJ, Brasil
}

\section{Descritores}

Transtornos mentais, epidemiologia\#.

Escalas de gradação psiquiátrica\# ${ }^{\#}$.

Estudos multicêntricos, métodos".

Inquéritos de morbidade\#.

Prevalência. Estudos

epidemiológicos. Entrevista

psiquiátrica padronizada. - Estudos de avaliação.

\section{Resumo}

\section{Objetivo}

Discutir aspectos metodológicos da estratégia de identificação de casos psiquiátricos, em duas etapas, em estudos epidemiológicos.

\section{Métodos}

Analisa-se a metodologia utilizada no Estudo Multicêntrico de Morbidade Psiquiátrica do Adulto realizado em três cidades brasileiras, entre 1990-1991. Na primeira etapa do citado estudo, uma amostra aleatória (6.740 indivíduos) da população foi selecionada e submetida a um rastreamento com o Questionário de Morbidade Psiquiátrica do Adulto - QMPA. Na segunda etapa, uma subamostra (775 indivíduos) foi selecionada e submetida ao Inventário de Sintomas do DSMIII (Diagnostic and Statistical Manual of Mental Disorders), aplicado por psiquiatras e psicólogos treinados, para confirmação-diagnóstica.

Resultados

São descritos os procedimentos empregados para a estimativa das prevalências, mostrando que o fraco desempenho da escala de rastreamento não compromete o método.

Conclusão

A vantagem da metodologia é a de corrigir as distorções apresentadas pelos instrumentos atuais de identificação de casos psiquiátricos.

\author{
Abstract \\ Objective \\ To discuss methodological aspects of the two stages in the identification of psychiatric \\ cases in epidemiological studies. \\ Methods \\ Analyze the methodology used in the Multicentric Psychiatric Morbidity Study, which \\ was conducted in three Brazilian cities (São Paulo, Brasília and Porto Alegre). In \\ the first stage of that study, a random sample (6,740 individuals) of the population
}

Correspondência para/Correspondence to: Sérgio Baxter Andreoli

Núcleo de Estatística e Metodologia Aplicadas Rua Dr. Bacelar, 334, Vila Clementino

04023-900 São Paulo, SP, Brasil

E-mail: andreoli@psiquiatria.epm.br
*Estudo financiado pela Fapesp (Processo no 94/0526-0).

Edição subvencionada pela Fapesp (Processo no 00/01601-8)

Recebido em 17/6/1999. Reapresentado em 19/4/2000. Aprovado em 4/5/2000. 
was drawn and all the participants were screened with the Questionnaire of Psychiatric Morbidity of the Adult (QMPA). In the second stage, a sample (775 individuals) of this population was drawn and these individuals were submitted to the Inventory of Symptoms of DSM-III, carried out by psychiatrists and trained psychologists.

\section{Results}

The study procedure for estimating the prevalence is described in details, showing that though the screening scales are a weak tool, they don't interfere with the methodology.

Conclusion

The advantage of this methodology is to correct any distortions caused by the current tools used in the identification of psychiatric cases.

\section{INTRO DUÇÃO}

O objetivo do presente estudo é avaliar a aplicabilidade dos métodos de identificação de casos psiquiátricos, em amostras populacionais, utilizando procedimentos de rastreamento, com base nos procedimentos e resultados da análise dos dados do Estudo Multicêntrico de Morbidade Psiquiátrica do Adulto, ${ }^{1}$ conduzido em três cidades brasileiras, entre 1990-1991. Nesse sentido, são apresentados, em detalhe, as etapas e os procedimentos empregados no citado estudo para as estimativas de prevalência, procurando demonstrar que o desempenho de escalas de rastreamento, caso corretamente avaliado em termos de sensibilidade, especificidade e valor preditivo, não compromete necessariamente a exeqüibilidade do método.

Os inquéritos populacionais são estudos epidemiológicos que se baseiam em um número grande de entrevistas domiciliares. Em estudos de morbidade psiquiátrica, a entrevista-diagnóstica realizada por psiquiatras é pouco indicada por se tratar de procedimento caro e de reduzida confiabilidade. A utilização de procedimentos padronizados de identificação de caso, portanto, constitui uma forma de baratear os custos e, assim, viabilizar tais estudos, aumentando sua confiabilidade. A década de 70 foi marcada pelo desenvolvimento de procedimentos dessa natureza, resultando na construção de série de instrumentos padronizados, destinados a auxiliar tanto o diagnóstico psiquiátrico na clínica como a identificação de casos em estudos epidemiológicos.

Os instrumentos padronizados podem ser classificados em entrevistas semi-estruturadas, entrevistas totalmente estruturadas e escalas de rastreamento. As entrevistas semi-estruturadas são compostas por listas de sintomas psiquiátricos elaboradas a partir de observações de pacientes da clínica; em geral, são desenhadas para serem utilizadas por clínicos habilitados. As entrevistas totalmente estruturadas também são compostas por listas de sintomas psiquiátricos, porém a presença ou a ausência do sintoma é estabelecida por intermédio de uma árvore de decisões totalmente estruturada; são preferencialmente administradas por entrevistadores leigos.

As escalas de rastreamento foram os primeiros instrumentos padronizados utilizados nos levantamentos epidemiológicos. Contêm questões que se referem a queixas psicológicas, psicossomáticas e fisiológicas encontradas nos transtornos psiquiátricos e podem ser auto-aplicadas ou aplicadas por entrevistadores leigos. Ao indivíduo é atribuído um escore total, resultado da soma das respostas positivas. Para cada escala, calcula-se um ponto de corte que melhor discrimina os indivíduos "doentes" dos "não-doentes".

Até o início da década de 70, existia uma pequena família dessas escalas breves de rastreamento, muito parecidas no conteúdo, que foram utilizadas em aproximadamente um terço dos estudos epidemiológicos realizados no período para medir construtos como: "saúde mental", "transtorno psiquiátrico", "doença mental", "ajuste emocional", "sintomas de estresse" e "sintomas psicofisiológicos". Não existia, nessa época, uma preocupação dos pesquisadores com uma melhor discriminação dos casos identificados, o que tornava os primeiros estudos epidemiológicos muito genéricos quanto ao diagnóstico de doença mental. ${ }^{14}$ As taxas de prevalência de doença mental eram calculadas tomando como base o escore do instrumento que melhor discriminava casos de não-casos.

A partir da década de 70, houve uma mudança na posição dos psiquiatras com relação à importância do diagnóstico, levando, por exemplo, ao desenvolvimento de sistemas classificatórios multiaxiais como o DSM-III. ${ }^{2}$ A partir daí, o esforço de desenvolvimento de instrumentos de identificação de casos concentrouse em métodos capazes de melhor discriminar o diagnóstico psiquiátrico. As escalas de rastreamento, den- 
tro desse contexto, passaram a ser desenvolvidas e utilizadas como indicadoras de casos potenciais e não como instrumento diagnóstico individual. A aplicação desse tipo de instrumento em uma primeira etapa de pesquisa, em conjunto com instrumentos estruturados, dentro de um levantamento epidemiológico, caracteriza a metodologia dos chamados desenhos multifásicos.

Levantamento populacional conduzido com esse método oferece vantagens consideráveis: apresenta um custo relativamente baixo em um tempo curto, pois a grande maioria das entrevistas é feita por entrevistadores leigos, com um instrumento de fácil aplicação, e apenas uma proporção pequena de casos precisam ser submetidos a entrevista com psiquiatra. É possível um treinamento eficiente de clínicos na aplicação da entrevista padronizada, e a natureza diagnóstica, bem como a quantidade de transtornos específicos de morbidade, podem ser determinados dentro de limites de confiança aceitáveis. ${ }^{9}$ Muitos levantamentos populacionais têm sido conduzidos utilizando estratégia similar para a identificação de casos, sendo o estudo brasileiro um exemplo.

\section{MÉTODOS}

\section{Aspectos do estudo multicêntrico}

O Estudo Multicêntrico de Morbidade Psiquiátri$\mathrm{ca}^{1}$ é o primeiro estudo epidemiológico de transtornos psiquiátricos realizado no Brasil que utiliza metodologia de identificação de caso em duas etapas, com instrumentos padronizados. O objetivo desse estudo foi estimar a prevalência de morbidade psiquiátrica em amostras representativas da população acima de 15 anos, residentes em três áreas metropolitanas brasileiras (Brasília, São Paulo e Porto Alegre). Para a identificação de casos psiquiátricos, foram utilizadas duas escalas psiquiátricas aplicadas em duas etapas (Figura). Na primeira etapa, o Questionário de Morbidade Psiquiátrica do Adulto (QMPA) foi aplicado para rastreamento de casos psiquiátricos; esses indivíduos compõem o que passará a chamar de amostra. Na segunda fase, $30 \%$ de prováveis casos e $10 \%$ de prováveis não-casos, identificados pelo QMPA, foram selecionados e submetidos ao inventário de sintomas do DSM-III; esse subgrupo de indivíduos compõe o que passará a chamar de subamostra.

\section{Plano amostral}

A amostragem realizada em Brasília foi estratificada geograficamente, tendo como estratos o plano piloto e as cidades satélites. De acordo com a distribuição demográfica, os estratos foram subdivididos em

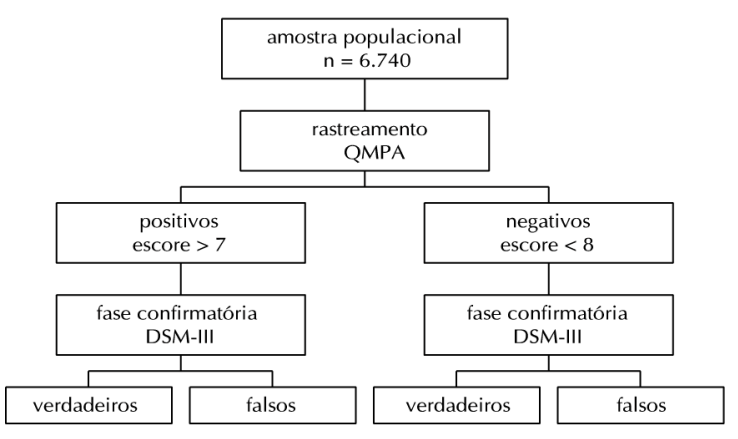

Figura - Levantamento populacional com rastreamento.

setores, segundo a situação socioeconômica da população. Dentro de cada setor, o tamanho da amostra de domicílios foi proporcional ao tamanho da sua população. Com base em um mapeamento atualizado, foi sorteado um domicílio como ponto de partida para cada entrevistador, que prosseguia incluindo na amostra a residência de número imediatamente superior, até esgotar o número de questionários previamente determinado pelos supervisores de campo. O tamanho da amostra foi calculado em 741 residências, tendo como base a média de 2,7 pessoas maiores de 15 anos por domicílio em Brasília. No total foram obtidas informações de 2.347 sujeitos.

Em São Paulo, a amostragem foi estratificada por condição socioeconômica. A cidade é composta por 48 distritos, dos quais 3 foram selecionados com base nos indicadores de saúde como representativos de toda a população. Assim, foram selecionados os distritos de Brasilândia, Vila Guilherme e Aclimação para as categorias socioeconômicas inferior, média e superior. A amostragem foi composta por $56,1 \%$ da população de Brasilândia (146 setores censitários), 27,2\% de Vila Guilherme (65 setores censitários) e 16,7\% da Aclimação (63 setores censitários). Foram selecionados 30 setores censitários para sorteio de 20 domicílios por setor (17 de Brasilândia, 8 de Vila Guilherme e 5 de Aclimação). Foram substituídas 93 famílias (15,5\% da amostra): 33 na Brasilândia (6 recusas e 27 ausências), 28 na Vila Guilherme (4 recusas e 24 ausências) e 32 na Aclimação (16 recusas e 16 ausentes). A amostra efetivamente pesquisada foi de 620 residências, totalizando uma amostra de 1.742 sujeitos maiores de 15 anos.

Em Porto Alegre, a Fundação Metropolitana de Desenvolvimento (Metroplan) identifica 96 zonas de tráfego (ZT). Cada ZT engloba 740 setores censitários, com uma média de 350 unidades habitacionais e 1.750 habitantes em cada setor. Cada ZT foi ordenada, segundo a renda média em 1986, em 10 estratos socioeconômicos. De cada um dos estratos foram sorteados 3 domicílios localizados na 
lista de domicílios por setor, atualizada para o censo de 1991. Cada um desses domicílios foi o identificador do setor censitário que passou a ser o conglomerado da investigação. Dentro de cada um desses setores censitários foram sorteados 37 domicílios, sendo 30 para a amostra e 7 para eventuais reposições. A amostra efetivamente pesquisada foi de 998 residências, com 903 famílias concordando em participar do estudo, o que resultou numa perda de $9,5 \%$. No total foram obtidas as informações de 2.384 sujeitos.

\section{Instrumentos}

Questionário de Morbidade Psiquiátrica do Adulto (QMPA). Trata-se de uma escala de rastreamento desenvolvida no Brasil por Santana ${ }^{13}$ com 45 questões, na sua última versão. As questões do QMPA abrangem os mais freqüentes sinais e sintomas característicos das doenças mentais, além de questões sobre tratamento psiquiátrico, uso anterior e atual de drogas psicofarmacológicas. O QMPA pode ser auto-aplicado ou aplicado por entrevistador leigo e pode ser respondido por terceiros. As alternativas de respostas são sim ou não, às quais são atribuídos valores 1 ou 0 . As primeiras 43 questões devem ser respondidas sobre o próprio indivíduo do qual se quer saber o estado de saúde mental, e as duas últimas questões (questões 44 e 45) referem-se à sintomatologia apresentada por qualquer um dos membros da família do indivíduo do qual se quer saber o estado de saúde mental.

Santana ${ }^{13}$ validou o QMPA realizando um estudo-piloto e um teste de campo. Em ambos, os escores obtidos pelo QMPA foram confrontados com uma entrevista psiquiátrica semi-estruturada utilizada para a definição de caso, tendo como critério diagnóstico a Classificação Internacional de Doenças - versão 8 (CID-8). O QMPA, para o ponto de corte $6 / 7$, apresentou uma sensibilidade de $75 \%$ no estudo-piloto e $93 \%$ no teste de campo e uma especificidade de $53 \%$ e $94 \%$. Estudou, também, a confiabilidade do QMPA que apresentou um Kappa ponderado de 0,88 .

Inventário de Sintomas do DSM-III. O inventário foi desenvolvido no Departamento de Psiquiatria da Universidade de Washington, St. Louis, EUA, para verificar os diagnósticos gerados pelo Diagnostic Interview Schedule (DIS). ${ }^{12}$ Investiga 39 diagnósticos do DSM-III ${ }^{2}$ e pode funcionar como um padrão válido para a avaliação de sintomas psiquiátricos e, conseqüentemente, identificação de caso psiquiátrico, desde que aplicado por clínicos treinados e familiarizados com os critérios do DSM-
III. ${ }^{8}$ Todos os diagnósticos que aparecem no instrumento são definidos e têm seus critérios estabelecidos no DSM-III. Para alguns dos diagnósticos, o instrumento apresenta uma lista de sintomas, e para ser classificado como positivo em um ou mais de um diagnóstico, o indivíduo deve apresentar um número mínimo de sintomas e preencher critérios definidos no DSM-III, tais como: pertencer a uma dada faixa de idade determinada, ter apresentado tais sintomas durante um período de tempo determinado, não apresentar outro diagnóstico que exclua a sua presença ou qualquer outra condição de exclusão.

Para a codificação dos sintomas são utilizados 3 níveis: (1) o sintoma está ausente ou não há evidência clínica de ser relevante; (2) o sintoma está presente e é relevante do ponto de vista clínico psiquiátrico; (3) incerteza na distinção entre presença e ausência do sintoma. Para a codificação-diagnóstica são utilizados 4 níveis: (1) ausente; (2) preenche os critérios do DSM-III e não é excluído por outro diagnóstico do DSM-III; (3) preenche os critérios do DSM-III somente se as regras de exclusão forem ignoradas; (4) incerto. Todos os diagnósticos são feitos tomando como base o tempo de vida do entrevistado; assim, para os indivíduos que preenchem os critérios para um diagnóstico, o período de aparecimento desse é codificado em: (1) nas últimas duas semanas; (2) de duas a menos de um mês; (3) de um mês a 6 meses; (4) de 6 meses a um ano; (5) menos de um ano (não sabe quando); (6) mais de um ano atrás.

O inventário de sintomas do DSM-III está organizado para cada diagnóstico de formas diferentes. Existem diagnósticos sem lista e com lista de sintomas e critérios, sendo que esses últimos podem apresentar a lista de sintomas hierarquizada ou não. A hierarquização, nesse caso, significa que um ou mais sintomas devem ser preenchidos de modo afirmativo para que um grupo subseqüente de sintomas seja investigado. Quando não hierarquizado, o entrevistador deve investigar todos os sintomas do diagnóstico. No caso dos diagnósticos sem lista de sintomas e critérios, o entrevistador deve indicar o código diagnóstico de acordo com os critérios da classificação-diagnóstica do DSM-III.

Para o caso em que o indivíduo preenche os critérios para um diagnóstico e o período de aparecimento foi no último ano (codificação de tempo 1 ou 2), o entrevistado deve responder se esteve incomodado por esses sintomas a maior parte do tempo: (1) não; (2) sim. Esse indicador, originalmente ausente do inventário de sintomas do DSM-III, foi introduzido para uma avaliação da gravidade do quadro apresentado pelo paciente. 


\section{Procedimentos do estudo de campo}

Sorteados os domicílios, as famílias foram procuradas pelos entrevistadores, em sua maioria estudantes de medicina ou de outras profissões de saúde, previamente treinados para aplicar o QMPA. Quando existia dúvida se o endereço sorteado havia se alterado (por exemplo: em caso de demolição ou situação de abandono) eram feitas até 3 tentativas pelos entrevistadores antes das famílias serem substituídas. No domicílio, o entrevistador solicitava a presença da dona da casa ou de pessoa adulta e adequada a prestar as informações (informante-chave). Esse informante-chave respondia às questões do QMPA, também para os outros membros da família, maiores de 15 anos.

Com um intervalo que variou entre um e quatro semanas da primeira etapa do estudo, uma subamostra de indivíduos foi entrevistada por meio do inventário de sintomas do DSM-III. O estudo empregou um total de 25 psiquiatras e psicólogos com treinamento clínico para a condução da fase de confirmação diagnóstica. Esses profissionais foram treinados para aplicar o inventário de sintomas do DSM-III por meio de cursos sobre os critérios diagnósticos, vídeo-tapes de entrevistas psiquiátricas e entrevistas supervisionadas. Os resultados do exame de confirmação diagnóstica eram registrados de acordo com o nível de certeza da presença de patologia, duração do transtorno e grau de gravidade. A gravidade foi avaliada pelos próprios entrevistados, nos casos em que o entrevistado havia preenchido os critérios para qualquer um dos diagnósticos no último mês.

\section{Técnica de cálculo das estimativas de prevalência}

\section{Estimativas globais}

Estimativas globais de prevalência foram calculadas para toda a vida dos indivíduos (lifetime prevalence). Os cálculos foram realizados pela adequação da capacidade de identificação de caso do QMPA, tomando como padrão o diagnóstico feito por meio do inventário de sintomas do DSM-III. Para tanto, foram calculados, na subamostra, os in- dicadores de validade do QMPA (sensibilidade, especificidade, valor preditivo positivo e valor preditivo negativo), com o ponto de corte $7 / 8$, utilizando como padrão de comparação a subamostra com qualquer dos 39 diagnósticos do DSM-III, excetuando tabagismo.

Na Tabela 1, e conforme já analisado em outra publicação, ${ }^{5}$ o desempenho do QMPA mostrou-se abaixo do observado em estudos de validação anteriores, com proporções de classificação incorreta acima de $33 \%$ em todas as subamostras. Os valores de sensibilidade ficaram em torno de $50 \%$ e os valores de especificidade variaram de $70 \%$ a $80 \%$, mostrando um desempenho na direção contrária ao esperado para um instrumento de rastreamento, qual seja, o aumento da proporção de casos na subamostra.

Os valores preditivos positivos foram diferentes entre as cidades, apresentando-se adequados nas cidades de Brasília (83\%) e Porto Alegre (70\%), porém reduzidos na cidade de São Paulo (abaixo de 50\%). O valor preditivo negativo, ao contrário, apresentou valores insatisfatórios nas cidades de Brasília (53\%) e Porto Alegre (62\%), sendo mais adequado em São Paulo (72\%).

O valor preditivo positivo foi utilizado para as estimativas de prevalência global na amostra, pois permite calcular a proporção de indivíduos positivos no QMPA que possuem algum diagnóstico do DSMIII. Essas estimativas foram corrigidas pelo inverso do valor preditivo negativo, segundo a equação de Fleiss, ${ }^{7}$ em que Pe é a estimativa de prevalência, ps é a proporção de supostos casos identificados pelo QMPA na amostra, pn é a proporção de não suspeitos, VP+ e VP- são os valores preditivos do QMPA, conforme segue:

$$
\mathrm{Pe}=\mathrm{ps}(\mathrm{VP}+)+\mathrm{pn}(1-\mathrm{VP}-)
$$

O erro-padrão (EP) das estimativas de prevalência globais foram calculadas de acordo com a seguinte equação:

$$
E P(P e)=\sqrt{\frac{P e(1-P e)}{N}\left(1+(1-K) \frac{N-n}{n}\right.},
$$

Tabela 1 - Indicadores de desempenho do QMPA com o inventário de sintomas do DSM-III, distribuídos por gênero em três capitais brasileiras. Brasil, 1991.

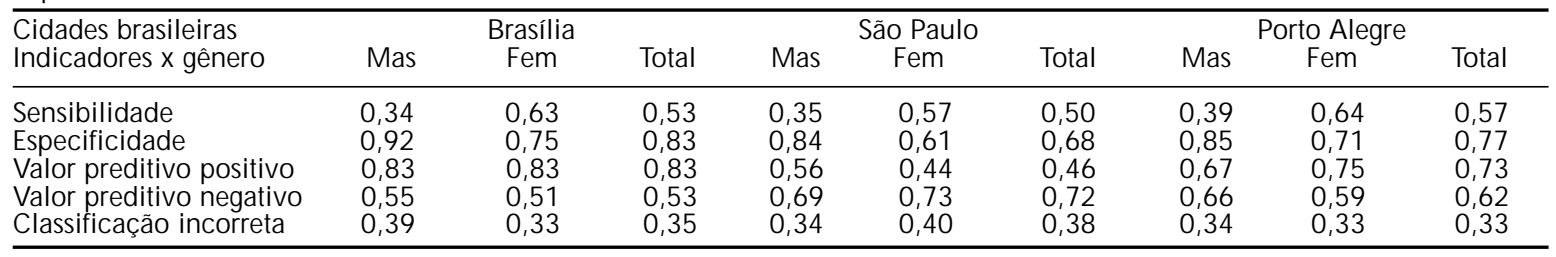

Q MPA - Q uestionário de Morbidade Psiquiátrica do Adulto DSM -III (Diagnostic and Statistical Manual of M ental Disorders) 
onde

$$
K=\frac{1-p s}{p s} \times \frac{(P e-(1-V P-))}{P e(1-P e)},
$$

em que Pe é a estimativa global de prevalência, ps é a prevalência de supostos casos na amostra, N é o número de indivíduos entrevistados na amostra, $\mathrm{n}$ é o número de indivíduos entrevistados na subamostra e VP - é o valor preditivo negativo.

Os resultados dos cálculos das estimativas de prevalência globais são mostrados na Tabela 2, em que se pode observar as proporções de supostos casos identificados pelo QMPA na amostra. As proporções apresentam-se com valores maiores para as mulheres, com aproximadamente o dobro daqueles apresentados pelos homens, nas três cidades estudadas. A cidade de São Paulo, em termos absolutos, apresenta as proporções maiores de casos supostos, entretanto, após a correção pelo desempenho do QMPA, a estimativa de prevalência global é menor do que aquelas apresentadas nas outras cidades.

A Tabela 3 mostra os parâmetros para os cálculos do erro-padrão, cuja estimativa de prevalência global foi de aproximadamente $0,03 \%$ para as três cidades. Quando distribuído por gênero, entretanto, as estimativas variaram de $0,04 \%$ a $0,06 \%$, com valores maiores para as mulheres nas cidades de São Paulo e Porto Alegre.

\section{Estimativas por diagnóstico}

As estimativas de prevalência por grupo-diagnóstico foram calculadas considerando as proporções de cada grupo-diagnóstico na subamostra de casos confirmados, multiplicadas pela prevalência global, de acordo com a seguinte fórmula:

$$
\mathrm{Pd}=\mathrm{Pe}(\mathrm{Fd} / \mathrm{Tc})
$$

em que Pd é a estimativa de prevalência do grupodiagnóstico, Pe a estimativa de prevalência global (Tabela 2), Fd é a frequiência de casos confirmados do diagnóstico e Tc corresponde ao total de indivíduos confirmados como tendo pelo menos um diagnóstico do DSM-III, identificados na segunda etapa do estudo.

A Tabela 4 mostra as freqüências de cada grupodiagnóstico de acordo com gênero e cidade, e observa-se que a soma das freqüências é maior do que o número de indivíduos, isto porque, segundo a metodologia adotada, os indivíduos podiam ter mais do que um diagnóstico. A Tabela 5 mostra os resultados da aplicação da fórmula acima para o cálculo das estimativas de prevalência por grupo-diagnóstico.

\section{DISCUSSÃO METODOLÓGICA}

A identificação de casos psiquiátricos em estudos epidemiológicos, envolvendo uma amostragem randômica da população, necessita de uma etapa de rastreamento antes do exame psiquiátrico. No exemplo do estudo multicêntrico brasileiro, esse procedimento não é simples por causa do desempenho insatisfatório das escalas de rastreamento. ${ }^{5}$ Mesmo assim, é possível calcular as estimativas de prevalência e seus erros-padrão sem prejuízo para a comparação dos resultados, tanto entre cidades estudadas quanto com os resultados de levantamentos populacionais que empregaram metodologia similar. ${ }^{1}$

As razões que melhor justificam a utilização de técnicas de rastreamento para a identificação de casos

Tabela 2 - Cálculo das estimativas de prevalência globais, utilizando as proporções de indivíduos supostos; casos identificados pelo Q M PA (>7) na amostra da população de três cidades brasileiras $(n=6.740)$, balanceadas pelos valores preditivos do Q M PA

\begin{tabular}{|c|c|c|c|c|c|c|c|c|c|}
\hline \multirow{2}{*}{$\begin{array}{l}\text { Cidades brasileiras } \\
\text { Parâmetros para o cálculo da prevalência }\end{array}$} & \multicolumn{3}{|c|}{ Brasília } & \multicolumn{3}{|c|}{ São Paulo } & \multicolumn{3}{|c|}{ Porto Alegre } \\
\hline & Masc & Fem & Total & Masc & Fem & Total & Masc & Fem & Total \\
\hline $\begin{array}{l}\text { Supostos casos } \\
\text { Supostos não casos } \\
\text { Valor preditivo positivo } \\
\text { Valor preditivo negativo }\end{array}$ & $\begin{array}{l}0,07 \\
0,93 \\
0,83 \\
0,55\end{array}$ & $\begin{array}{l}0,16 \\
0,84 \\
0,83 \\
0,51\end{array}$ & $\begin{array}{l}0,12 \\
0,88 \\
0,83 \\
0,53\end{array}$ & $\begin{array}{l}0,12 \\
0,88 \\
0,56 \\
0,69\end{array}$ & $\begin{array}{l}0,26 \\
0,75 \\
0,44 \\
0,73\end{array}$ & $\begin{array}{l}0,18 \\
0,82 \\
0,46 \\
0,72\end{array}$ & $\begin{array}{l}0,07 \\
0,93 \\
0,67 \\
0,66\end{array}$ & $\begin{array}{l}0,18 \\
0,81 \\
0,75 \\
0,59\end{array}$ & $\begin{array}{l}0,13 \\
0,87 \\
0,73 \\
0,62\end{array}$ \\
\hline Estimativa de prevalência global & 0,47 & 0,54 & 0,51 & 0,34 & 0,31 & 0,31 & 0,36 & 0,47 & 0,43 \\
\hline
\end{tabular}
distribuídos por gênero. Brasil, 1991.

Tabela 3 - Cálculo dos desvios-padrão das estimativas de prevalência globais para as três cidades brasileiras distribuídos por

\begin{tabular}{|c|c|c|c|c|c|c|c|c|c|}
\hline \multirow{2}{*}{$\begin{array}{l}\text { Cidades brasileiras } \\
\text { Parâmetros para o cálculo de erro-padrão }\end{array}$} & \multirow{2}{*}{\multicolumn{2}{|c|}{$\begin{array}{l}\text { Brasília } \\
\text { Fem }\end{array}$}} & \multirow[b]{2}{*}{ Total } & \multirow{2}{*}{\multicolumn{2}{|c|}{$\begin{array}{l}\text { São Paulo } \\
\text { Fem }\end{array}$}} & \multirow[b]{2}{*}{ Total } & \multicolumn{3}{|c|}{ Porto Alegre } \\
\hline & & & & & & & Masc & Fem & total \\
\hline $\begin{array}{l}\text { Estimativa de prevalência global } \\
\text { Supostos casos } \\
\text { Valor preditivo negativo } \\
\text { Número de indivíduos na amostra } \\
\text { Número de indivíduos na subamostra }\end{array}$ & $\begin{array}{l}0,47 \\
0,07 \\
0,55 \\
1.086 \\
106\end{array}$ & $\begin{array}{c}0,54 \\
0,16 \\
0,51 \\
1.258 \\
168\end{array}$ & $\begin{array}{l}0,51 \\
0,12 \\
0,53 \\
2.344 \\
274\end{array}$ & $\begin{array}{l}0,34 \\
0,12 \\
0,69 \\
845 \\
71\end{array}$ & $\begin{array}{l}0,31 \\
0,26 \\
0,73 \\
897 \\
151\end{array}$ & $\begin{array}{c}0,31 \\
0,18 \\
0,72 \\
1.742 \\
222\end{array}$ & $\begin{array}{l}0,36 \\
0,07 \\
0,66 \\
1.075 \\
97\end{array}$ & $\begin{array}{c}0,47 \\
0,18 \\
0,59 \\
1.309 \\
182\end{array}$ & $\begin{array}{c}0,43 \\
0,13 \\
0,62 \\
2.384 \\
279\end{array}$ \\
\hline Erro-padrão & 0,04 & 0,04 & 0,03 & 0,06 & 0,03 & 0,03 & 0,05 & 0,04 & 0,03 \\
\hline
\end{tabular}
gênero. Brasil, 1991. 
Tabela 4 - Freqüência dos grupos-diagnóstico na subamostra de três cidades brasileiras distribuídos por gênero. Brasil, 1991.

\begin{tabular}{|c|c|c|c|c|c|c|}
\hline \multirow[b]{2}{*}{ Diagnósticos do DSM-III } & \multicolumn{2}{|c|}{ Brasília } & \multicolumn{2}{|c|}{ São Paulo } & \multicolumn{2}{|c|}{ Porto Alegre } \\
\hline & Masc & Fem & Masc & Fem & Masc & Fem \\
\hline Síndrome cerebrorgânica & 2 & 2 & 0 & 0 & 2 & 4 \\
\hline Abuso e dependência de álcool & 18 & 2 & 12 & 0 & 19 & 5 \\
\hline Abuso e dependência de drogas & 0 & 1 & 1 & 0 & 3 & 7 \\
\hline Esquizofrenias & 0 & 0 & 0 & 1 & 2 & 2 \\
\hline Transtornos afetivos - depressão & 3 & 8 & 0 & 7 & 7 & 30 \\
\hline Transtornos afetivos - mania e ciclotimias & 1 & 0 & 0 & 1 & 2 & 1 \\
\hline Transtornos delirantes, psicóticos e outros & 0 & 1 & 0 & 2 & 2 & 3 \\
\hline Transtornos conversivos e somatodissociativos & 4 & $2 \overline{7}$ & 1 & 8 & 2 & 17 \\
\hline Transtornos de pânico, ansiedade e outros & 16 & 45 & 6 & 25 & 6 & 29 \\
\hline Transtornos obsessivos compulsivos & 1 & 1 & 0 & 0 & 2 & 5 \\
\hline Fobias - agora, social e simples & 13 & 47 & 4 & 19 & 9 & 43 \\
\hline Transtorno de ajustamento & 3 & 4 & 0 & 2 & 2 & 3 \\
\hline Fatores psicológicos que afetam o físico & 13 & 18 & 8 & 11 & 3 & 9 \\
\hline Retardo mental & 4 & 6 & 3 & 3 & 3 & 9 \\
\hline Número de diagnósticos positivos & 78 & 162 & 35 & 79 & 64 & 167 \\
\hline Número de individuos com diagnóstico positivo & 56 & 111 & 26 & 52 & 41 & 105 \\
\hline
\end{tabular}

Tabela 5 - Estimativas de prevalência por grupo-diagnóstico em três cidades brasileiras distribuídos por gênero. Brasil, 1991.

\begin{tabular}{|c|c|c|c|c|c|c|}
\hline \multirow[b]{2}{*}{ Diagnósticos do DSM-III } & \multicolumn{2}{|c|}{ Brasília } & \multicolumn{2}{|c|}{ São Paulo } & \multicolumn{2}{|c|}{ Porto Alegre } \\
\hline & Masc & Fem & Masc & Fem & Masc & Fem \\
\hline Síndrome cerebrorgânica & 1,9 & 1,1 & & - & 1,7 & 2,0 \\
\hline Abuso e dependência de álcool & 15,0 & 1,1 & 15,2 & - & 16,0 & 2,5 \\
\hline Abuso e dependência de drogas & - & 0,5 & 1,3 & - & 2,4 & 3,5 \\
\hline Esquizofrenias & - & 0,0 & - & 0,6 & 1,7 & 1,0 \\
\hline Transtornos afetivos - depressão & 1,9 & 3,8 & - & 3,8 & 5,9 & 14,5 \\
\hline Transtornos afetivos - mania e ciclotimias & 0,9 & - & - & 0,6 & 1,7 & 0,5 \\
\hline Transtornos delirantes, psicóticos e outros & - & 0,5 & - & 1,2 & 1,7 & 1,5 \\
\hline Transtornos conversivos e somatodissociativos & 3,3 & 13,0 & 1,3 & 4,3 & 1,7 & 8,0 \\
\hline Transtornos de pânico, ansiedade e outros & 13,6 & 21,6 & 7,6 & 13,9 & 5,2 & 14,0 \\
\hline Transtornos obsessivos compulsivos & 0,9 & 0,5 & 0,0 & - & 1,7 & 2,5 \\
\hline Fobias - agora, social e simples & 10,8 & 22,7 & 4,9 & 10,4 & 7,7 & 20,5 \\
\hline Transtorno de ajustamento & 1,9 & 2,2 & - & 1,2 & 1,7 & 1,5 \\
\hline Fatores psicológicos que afetam o físico & 10,8 & 8,6 & 10,2 & 6,1 & 2,4 & 4,5 \\
\hline Retardo mental & 3,3 & 2,7 & 3,6 & 1,7 & 2,4 & 4,5 \\
\hline
\end{tabular}

em inquéritos populacionais são as seguintes: controlar o erro de identificação das escalas ${ }^{7}$ e reduzir o tamanho da amostra para posterior investigação. No estudo em pauta, a intenção, ao utilizar esse método, foi inicialmente reduzir o tamanho da amostra e aumentar a proporção de casos, entrevistando um número maior de supostos casos na etapa de confirmação-diagnóstica. Com a aplicação desse procedimento, a subamostra ficou com o tamanho de 775 indivíduos, e as proporções de casos com pelo menos um diagnóstico do DSM-III foram de $61 \%$ na cidade de Brasília, 35\% em São Paulo e 52\% em Porto Alegre.

O controle do erro de identificação do QMPA pode ser ilustrado quando se comparam as prevalências estimadas por meio dos valores preditivos positivos sem correção (ps x VP+) e as prevalências estimadas após a correção. Sem a correção, a prevalência foi de 9,9\% em Brasília, 8,3 \% em São Paulo e 9,5\% em Porto Alegre; podendo-se observar que existe entre elas uma pequena variação. Entretanto, quando corrigidas pelos falsos negativos (1-VP-), os valores das estimativas aumentam e mostram grande variação entre as cidades: $51 \%$ em Brasília, 31\% em São Paulo e $43 \%$ em Porto Alegre.

Dessa forma, as variações dos valores das estimativas foram devidas às proporções elevadas de falsos negativos (1- VP-) do QMPA, quais sejam: 47\% em Brasília, 28\% em São Paulo e 38\% em Porto Alegre. É bem conhecido na literatura que o indicador mais importante e crítico para uma escala de rastreamento é a proporção de falsos negativos ${ }^{6} \mathrm{e}$, no presente exemplo, não só desempenhou papel importante de controle de erro, mas também funcionou como importante moderador do exame psiquiátrico.

Esse papel moderador do exame psiquiátrico pode ser observado quando são comparadas as prevalências encontradas nas subamostras $(61 \% ; 35 \% ; 52 \%)$ e as prevalências estimadas $(51 \% ; 31 \% ; 43 \%)$. Embora seja uma comparação de dados brutos, no caso da subamostra, é ilustrativo observar que a redução relativa nas proporções foi de $16 \%, 11 \%$ e $17 \%$, portanto, uma atenuação diferenciada entre as cidades.

A observação desses resultados provoca reflexão acerca da utilização de técnicas de rastreamento em estudos epidemiológicos no campo da psiquiatria. As escalas são para esses estudos mais do que rastreadoras de caso psiquiátrico; são um fator de correção do erro-diagnóstico, e essa seria a maior justificativa para sua utilização.

De um modo geral, a identificação de caso psiquiátrico na pesquisa epidemiológica é ainda precária, pois está totalmente baseada em sintomas. Por esse 
motivo, não existem razões para acreditar que algum instrumento que se proponha a substituir o exame clínico possa ser definitivo nessa função. As críticas aos estudos que utilizam a etapa de rastreamento baseiam-se no fraco desempenho dos instrumentos de rastreamento. É preciso, entretanto, lembrar que o desempenho é sempre avaliado tomando como referência outros instrumentos, que por sua vez também terão problemas de validade e confiabilidade. Observando os resultados, é possível supor que, se por um lado a escala de rastreamento deixa de identificar casos, o inventário de sintomas do DSM-III, aplicado por um especialista, tende a identificar casos a mais. Ao confrontar os dois instrumentos, e efetuadas as devidas correções, as estimativas de prevalência podem efetivamente servir de base para as comparações entre as cidades.

O instrumento diagnóstico utilizado na segunda etapa do estudo multicêntrico, analisado no presente trabalho, mostrou-se adequado, mas possui o inconveniente de precisar de clínicos treinados para sua aplicação. Atualmente dispõe-se de uma versão estruturada do CIDI $^{15}$ (Composite International Diagnostic Interview) que investiga diagnósticos psiquiátricos baseados na Classificação Internacional de Doenças, versão 10. Além de utilizar uma clas-

\section{REFERÊNCIAS}

1. Almeida-Filho N, Mari JJ, Coutinho E, França JF, Fernandes J, Andreoli SB et al. Brazilian multicentric study of psychiatric morbidity: methodological feature and prevalence estimates. Br J Psychiatry 1997;171:524-9.

2. American Psychiatric Association. Diagnostic and statistical manual of mental disorders. 3rd ed. Washington (DC): 1980.

3. Andreoli SB, Mari JJ, Blay SL, Almeida Filho $\mathrm{N}$ de, Coutinho E, França JF et al. Estrutura fatorial do questionário de morbidade psiquiátrica de adultos aplicado em amostras populacionais de cidades brasileiras. Rev Saúde Pública 1994;28:249-60.

4. Andreoli SB. Estudo das manifestações psicopatológicas na população brasileira [Tese]. São Paulo: U niversidade Federal de São Paulo; 1997.

5. Andreoli SB, Blay SL, Mari JJ. Escalas de rastreamento de psicopatologia. Rev Psiquiatr Clín 1998;25:229-32.

6. Deming WE. An essay on screening, or on two-phase sampling, applied to survey of a community. Int Stat Rev 1977;45:29-37. sificação de doença atualizada, o CIDI pode ser aplicado por entrevistadores leigos treinados.*

O rastreamento é um procedimento desejável em estudos populacionais no campo da psiquiatria, no entanto, alguns cuidados devem ser tomados ao se replicar esse estudo, principalmente no que diz respeito à escolha dos instrumentos. As escalas de rastreamento para esse fim disponíveis na língua portuguesa e com estudos de desempenho são: o QMPA,${ }^{13}$ o General Health Questionnaire $\left(\mathrm{GHQ}^{10}\right)$ e o Self Report Questionnaire $\left(S_{R Q}^{11}\right)$. Desses, o QMPA e o SRQ são semelhantes na sua forma e conteúdo, e o GHQ apresenta algumas diferenças no conteúdo, mas a principal distinção entre elas encontra-se na forma de medida dos sintomas (escalas de Likert no caso do GHQ). O QMPA, entretanto, apresenta um número maior de estudos de desempenho em amostras populacionais brasileiras, ${ }^{3,4,13}$ fornecendo subsídios para o cálculo da amostra submetida à segunda etapa e para ajustes na aplicação em estudos futuros.

\section{AGRADECIMENTOS}

A Ellis D' Arrigo Busnello, Josimar F França, Jéferson G Fernandes, pela coordenação regional do estudo muticêntrico, e a Cláudio T Miranda, Sérgio L Blay e Marcos T Ferraz pelas críticas e pelos comentários.

7. Fleiss JL. The control of misclassification error. In: Statistical methods for rates and proportions. 2nd ed. New York: John Wiley; 1981.

8. Helzer JE, Robins LN, M cEvoy LT, Spitznagel EL, Stoltzman RK, Famer A et al. A comparison of clinical and diagnostic interview schedule diagnoses. Physician reexamination of lay-interviewed cases in the general population. Arch Gen Psychiatr 1985;42:657-66.

9. Henderson S, D uncan-Jones P, Byrne DG, Scott R, Adcock S. Psychiatric disorder in Canberra. A standardized study of prevalence. Acta Psychiatr Scand 1979;60:355-74.

10. Mari JJ, Williams P. A comparison of the validity of two psychiatric screening questionnaires (GHQ -12 and SRQ -20) in Brazil, using Relative O perating Characteristic (ROC) analysis. Psychol Med 1985;15:651-9.

11. Mari JJ, Blay SL, lacoponi E. Reliability of the Brazilian version of the clinical interview schedule. Bol O ficina Sanit Panamer 1986;100:77-83. 
12. Robins LN, Helzer JE, Croughan J, Ratcliff KS. The NIMH Diagnostic Interview Schedule: its history, characteristics, and validity. Arch Gen Psychiatr 1981;38:381-9.

13. Santana W. Estudo epidemiológico das doenças mentais em bairro de Salvador. Salvador: Instituto de Saúde da Bahia; 1982 (Série de Estudos em Saúde).

14. Weissman M M. Epidemiology overview. In: Hales RE, Frances Al, editors. American Psychiatric Association Annual Review. $6^{\text {th }}$ ed. Washington (DC): American Psychiatric Association; 1987. p. 574-85.
15. Wittchen HU, Robins LN, Cottler LB, Sartorius N, Burke JD, Regier D et al. Cross-cultural feasibility, reliability and sources of variance of the Composite International Diagnostic Interview (CIDI). Br J Psychiatry 1991;159:645-53. 